\title{
EchoGéo
}

$51 \mid 2020$

Territoires « cyclonés »

\section{Mortalité et cyclones en Guadeloupe (Antilles françaises)}

Apports d'un nouveau référentiel géo-historique (1635-2018)

Frédéric Leone, Samuel Battut, Victoria Bigot, Guilhem Cousin Thorez, Thomas Candela et Freddy Vinet

\section{QpenEdition}

Journals

Édition électronique

URL : https://journals.openedition.org/echogeo/18919

DOI : $10.4000 /$ echogeo.18919

ISSN : 1963-1197

Éditeur

Pôle de recherche pour l'organisation et la diffusion de l'information géographique (CNRS UMR 8586)

Référence électronique

Frédéric Leone, Samuel Battut, Victoria Bigot, Guilhem Cousin Thorez, Thomas Candela et Freddy Vinet, " Mortalité et cyclones en Guadeloupe (Antilles françaises) », EchoGéo [En ligne], 51 | 2020, mis en ligne le 25 avril 2020, consulté le 10 août 2021. URL : http://journals.openedition.org/echogeo/ 18919 ; DOI : https://doi.org/10.4000/echogeo.18919

Ce document a été généré automatiquement le 10 août 2021.

EchoGéo est mis à disposition selon les termes de la licence Creative Commons Attribution - Pas d'Utilisation Commerciale - Pas de Modification 4.0 International (CC BY-NC-ND) 


\section{Mortalité et cyclones en Guadeloupe (Antilles françaises)}

Apports d'un nouveau référentiel géo-historique (1635-2018)

Frédéric Leone, Samuel Battut, Victoria Bigot, Guilhem Cousin Thorez, Thomas Candela et Freddy Vinet

Cette recherche a été réalisée dans le cadre du projet C3AF, Changement Climatique et Conséquences dans les Antilles Françaises (WP5), et a été cofinancée par le PO-FEDER de Guadeloupe (2014-2020). Le catalogue historique et la base de données SIG sur la mortalité sont consultables en ligne sur le site du projet : https://c3af.univ-montp3.fr/ (rubrique Pour en savoir plus). Les auteurs tiennent à remercier Jérémy Desarthe de la CCR pour sa relecture du catalogue historique, le personnel des archives départementales de Guadeloupe, dont Monsieur Garnier, les agents administratifs des services d'état-civil de la plupart des communes de Guadeloupe, ainsi que Christophe Montout de l'antenne Antilles Guyane de Météo-France, et Jean-Claude Huc, pour le temps consacré et les ressources partagées avec passion.

\section{Introduction}

1 La France, et encore moins ses territoires d'Outre-mer, ne disposent de référentiel national historique, détaillé, actualisé et centralisé, permettant de comptabiliser les victimes des aléas naturels autres que celles dues aux avalanches de neige (BD ANENA, 2019). Les données de mortalité sont dispersées dans des catalogues historiques ou des bases de données disparates, ce qui n'en facilite pas l'exploitation à des fins épidémiologiques et scientifiques. On peut citer par exemple la base BD SISFrance (2019) sur les séismes historiques, et sa variante sur les tsunamis, la base BD RTM (2019) sur les «risques naturels en montagne", la base de Données Historiques sur les Inondations (BDHI, 2019), ou la BD CATNAT (2019) multi-événements naturels, mais qui contrairement aux précédentes est en partie payante. Cette dernière reproduit une très grande partie des informations fournies par la base internationale Emergency Events Database (BD EM-DAT, 2019). L'unique base de données structurée autour de la problématique de la mortalité en France, et adaptée à une analyse fine des 
circonstances et lieux de décès, a été développée par des chercheurs de l'Université Paul-Valéry Montpellier 3 pour les inondations et la submersion marine (Boissier, 2013). Dénommée «Vict-In », elle a été valorisée dans plusieurs publications (Boissier et Vinet, 2009 ; Boissier, 2013 ; Vinet et al., 2011, 2016 et 2017), et sa structure, tout comme ses analyses, ont guidé cet article en étendant son contenu à l'archipel de Guadeloupe.

2 En effet, la situation de la Guadeloupe, notamment au regard de l'intensité et de la fréquence des aléas hydro-climatiques et météo-marins qui s'y manifestent, mérite une attention particulière centrée sur les impacts humains de ces phénomènes, en premier lieu la mortalité. La mortalité demeure un indicateur de vulnérabilité de ces territoires et de leurs populations, qui fait écho à toutes les stratégies de réduction des risques fondées principalement sur la protection des personnes. Elle peut ainsi devenir un indicateur de suivi des politiques publiques en matière de gestion des risques, a priori traçable dans le temps et l'espace, contrairement à d'autres formes d'impacts, notamment économiques ou matériels, qui sont plus difficiles à reconstituer à partir des archives documentaires ou du fil de l'actualité, et qui échappent davantage à la mémoire collective. La mortalité comme expression de la vulnérabilité constitue ce que Boissier (2013) appelle un « angle mort épistémologique » en France, contrairement à d'autres pays où ces approches sont développées (French et al., 1983; Mooney, 1983 ; Coates, 1999 ; Jonkman, 2005 ; Jonkman et Kelman, 2005 ; Jonkman et al., 2009 ; Coates et al., 2014).

3 Aussi, cet article vise à produire un catalogue historique et harmonisé de l'activité cyclonique significative ayant impacté l'archipel de Guadeloupe, renseigné en particulier par la mortalité, et complété pour la période récente par une base de données géo-référencée sur les victimes. Ces deux nouveaux référentiels vont nous permettre d'analyser l'évolution, les déterminants et les conditions de décès, en particulier en période cyclonique. Ce travail propose également des leviers de prévention des risques naturels en Guadeloupe.

\section{Méthodologie}

\section{Zone d'étude}

La recherche a concerné principalement la Guadeloupe (Basse-Terre et Grande-Terre) et ses dépendances (Marie-Galante, La Désirade, Les Saintes) (illustration 1). Ce territoire des Antilles françaises a eu un passé colonial de 1635 à 1946, puis a accédé au statut de département français d'Outre-mer en 1946, pour devenir Département-Région d'Outre-mer. Le catalogue historique constitué inclut également la partie française de l'île de Saint-Martin et l'île de Saint-Barthélemy, collectivités d'Outre-mer depuis 2007, aussi appelées îles du Nord, et situées à environ $250 \mathrm{~km}$ au nord de la Guadeloupe. Tous ces territoires appartiennent au bassin cyclonique nord-atlantique, une des zones les plus actives en matière de cyclogenèse et très exposée aux effets du réchauffement climatique (Cantet et al., 2014 ; Pilon et al., 2017 ; Belmadani et al., 2017 ; Hoarau et al., dans ce numéro). La saison cyclonique 2017 a donné deux cyclones majeurs de catégorie 5 sur les Petites Antilles (Irma puis Maria), entrainant un bilan humain de 183 morts d'après la base internationale BD EM-DAT (2019) du CRED, mais en définitive très variable suivant les sources (Nicolas et al., 2018 ; Kishore et al., 2018 ; Guha-Sapir et 
Checchi, 2018). Côté français, le bilan officiel a été de onze morts à Saint-Martin (Irma) et de quatre morts en Guadeloupe (Maria).

Illustration 1- Situation de la Guadeloupe et des îles du Nord dans leur contexte cyclonique historique

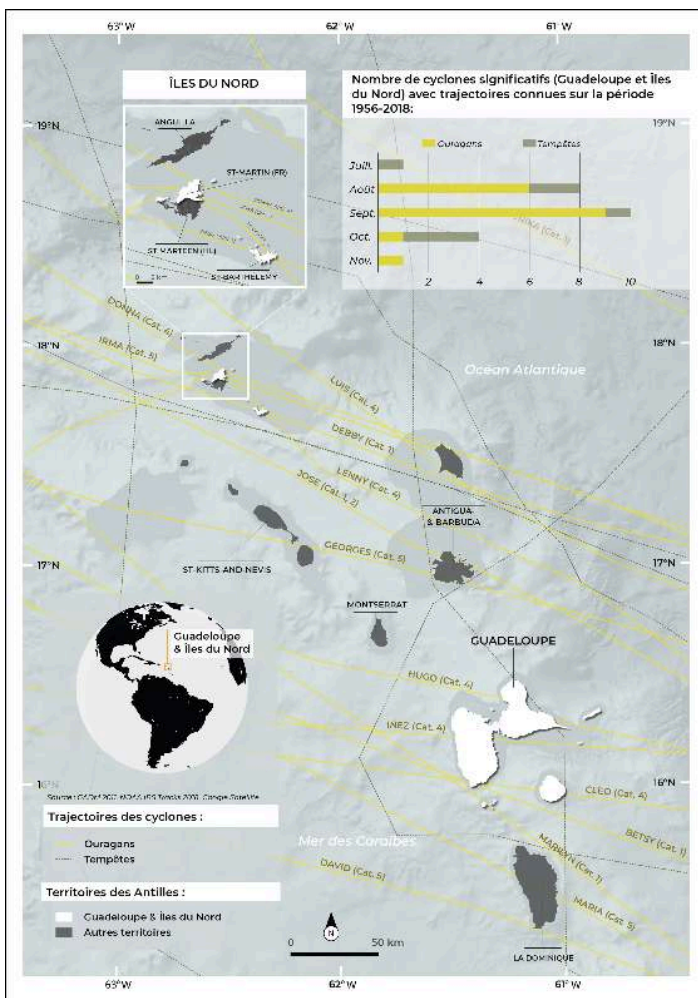

Sources: GADM 2011, NOAA IBTrACS 2018, Google Satellite. Auteurs : T. Candela et F. Leone.

\section{Critères de sélection}

Les événements hydro-climatiques étudiés regroupent les cyclones tropicaux, divisés en trois niveaux d'intensité (dépressions tropicales, tempêtes tropicales, ouragans), et leur cortège d'aléas induits que sont les inondations, la submersion marine et houles associées, les vents forts, la foudre et certains mouvements de terrain. Par le terme « significatif » on considère des événements ayant provoqué des dommages importants sur les territoires en question, et/ou entrainé au moins un décès. La notion de victime étant polysémique, donc particulièrement difficile à définir, c'est la définition de Jonkman et Kelman (2005) qui a été retenue pour la période récente (1950-2018), à savoir " a fatality that would not have occurred without a specific [...] event». Les victimes regroupent donc les décès directs (victimes touchées par l'aléa lors de sa manifestation) et les décès indirects (victimes affectées par un effet de l'aléa temporellement ou spatialement indépendant de sa manifestation), lorsqu'il est possible de clairement déterminer le lien entre l'aléa et le décès. Les blessés sont écartés de cette définition. Pour la période ancienne (1635-1949), principalement coloniale (1635-1946), la mortalité liée à des naufrages n'a pas été prise en compte à ce stade de nos recherches. 


\section{Catalogue historique des événements hydro-climatiques significatifs (CAT-EHC, 1635-2018)}

6 Plusieurs catalogues historiques permettent de reconstituer l'activité cyclonique aux Antilles et en Guadeloupe en particulier. On peut citer les bases de données en ligne de la NOAA (Rappaport et Fernandez-Partagas, 1997 ; Rappaport, 2000 ; Knapp et al., 2010), de l'association Infoclimat (BD Infoclimat, 2019), de l'Amicale des ouragans (2019), de la DEAL Guadeloupe (2008), les recherches historiques de Poey (1855), et les travaux de Caviedes (1991), Fernandez-Partagas et Diaz (1996), Landsea et al. (2004), Garcia-Herrera et al. (2005), Zahibo et al. (2009), Chenoweth (2006), Chenoweth et Divine (2008; 2012). Les travaux les plus complets menés par des historiens spécifiquement sur les Antilles françaises sont récents (Desarthe, 2014 ; Desarthe et Moncoulon, 2017 ; Garnier et al., 2017 ; Desarthe, dans ce numéro). Ils visent à analyser la sévérité des cyclones à travers les dommages, essentiellement matériels et économiques, ainsi que les réponses apportées au cours du temps par les sociétés antillaises. Ils peuvent être complétés par des études de cas, ou des retours d'expérience post-cycloniques, menés par exemple par Pagney (1991, 1998), Imbert et al. (1996), Candale (1999), Hamparian (1999) ou Sarant et al. (2003). Cependant, ces recherches historiques ou géographiques, ne décrivent que très rarement les bilans humains et encore moins les circonstances des décès.

7 Afin d'établir un nouveau référentiel sur les événements historiques significatifs en Guadeloupe (nommé CAT-EHC), il nous a fallu compiler et harmoniser ces catalogues existants, puis les enrichir avec de nouvelles informations issues de sources primaires reproduites (Saffache et al., 2003 ; Huc et Etna, 2015) ou originelles (archives nationales, presse locale, etc.). Ces recherches complémentaires ont notamment permis de compléter les bilans humains. La première partie du catalogue couvre principalement la période coloniale, et s'étend de 1635 à 1949. Les sources primaires consultées proviennent des archives Nationales d'Outre-Mer (ANOM) situées à Aix-en-Provence. Certains événements de cette partie du catalogue, mieux renseignés que d'autres, ont donné lieu à des bilans de mortalité spatialisés, soit par île, soit par commune. La seconde partie du catalogue porte sur la période dite récente, comprise entre 1950 et 2018. Elle a été composée à partir de sources locales, essentiellement la presse, disponibles en Guadeloupe, et d'enquêtes de terrain approfondies visant à retrouver les lieux de décès. Le CAT-EHC précise la date de l'événement, le type de phénomène, les territoires les plus impactés, la catégorie de l'ouragan, son nom international éventuel, le nombre de décès identifiés par d'autres auteurs (décès \#1), les sources correspondantes (en gras pour ce nombre de décès) et le nombre de décès identifiés au cours de notre propre recherche (décès \#2). Quand cela a été possible, les types et intensités des cyclones récents ont été renseignés au moyen de la base internationale IBTrACS de la NOAA (Knapp et al., 2010) et du site des pluies extrêmes de Météo France (2019). Le catalogue final recense 133 événements significatifs dont 45 avec des décès identifiés (tableau 1). 
Tableau 1 - Événements meurtriers issus du Catalogue historique des événements hydroclimatiques significatifs (Guadeloupe et dépendances, îles du Nord).

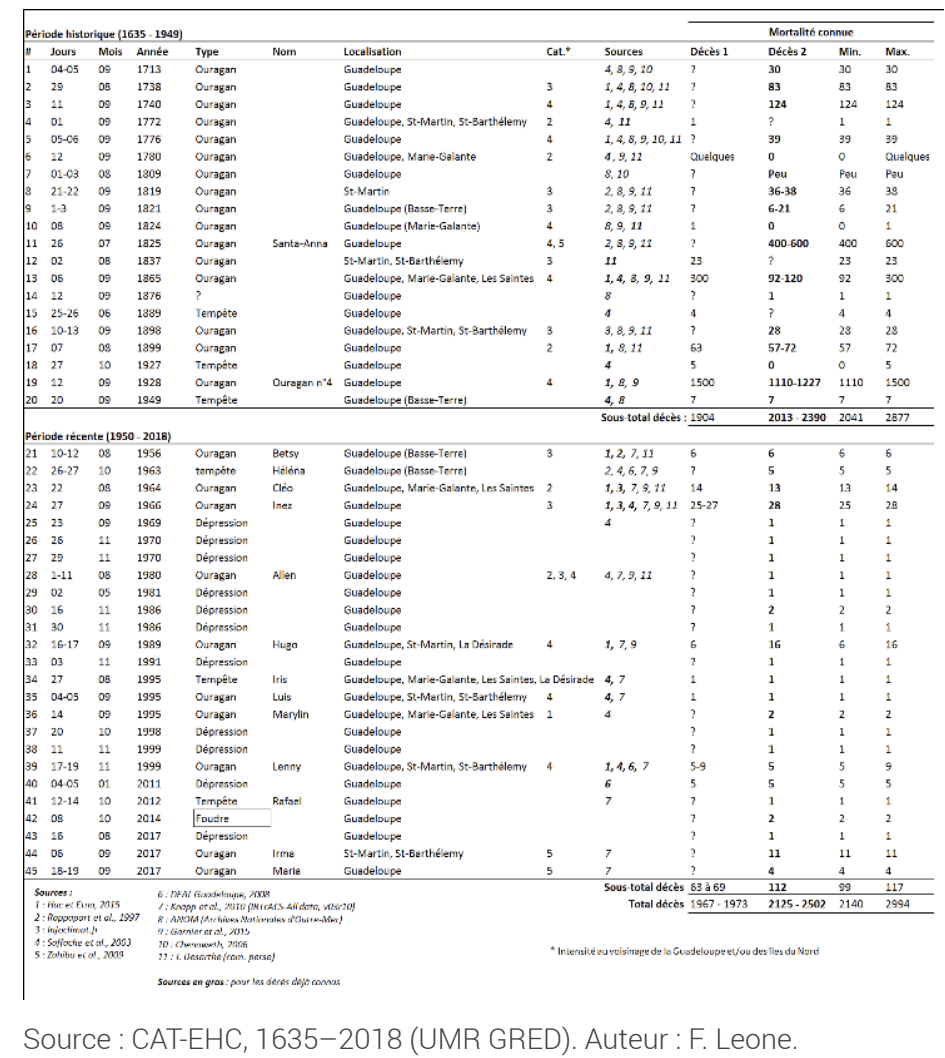

\section{Sources anciennes (1635-1949)}

$8 \mathrm{Au}$ cours du temps la Guadeloupe a été intégrée aux possessions françaises sous différents statuts. Les sources qui concernent la colonie sont conservées en grande partie aux ANOM, à Aix-en-Provence. On peut y distinguer les phénomènes d'envergure moyenne, n'affectant que la région de la Guadeloupe et ses dépendances, généralement appelés " coups de vent » ou parfois "bourrasques ", des phénomènes de plus grande envergure, qui affectent la région des Antilles, voire des Caraïbes, et qui sont désignés par les termes ouragans et cyclones. Le mot cyclone n'est cependant utilisé qu'à partir du XIX ${ }^{e}$ siècle, et concerne les cas les plus extrêmes (cyclone du 7 août 1899, cyclone du 12 septembre 1928). Nos recherches se sont concentrées sur 33 événements importants, dont 14 avec des bilans humains renseignés $(1713,1738,1740,1776,1809,1819,1821$, $1825,1865,1876,1898,1899,1928,1949)$. Les types de documents qui prédominent sont les rapports de gouverneurs et autres administrés, auxquels sont joints des états des pertes matérielles. Jusqu'au début du XIX siècle l'information est rare et les documents s'attachent à décrire surtout, et avec minutie, l'ampleur des pertes matérielles, tout en négligeant tout ou partie des pertes humaines. La presse de l'époque a également été d'une grande aide. Parce qu'on cherche à capter l'attention du lecteur et à mettre en avant les aspects spectaculaires des sinistres, il y est parfois question des victimes. Certains documents se sont avérés inexploitables pour notre sujet, comme les relevés en provenance des hôpitaux de l'île. La qualité de certaines sources a cependant permis d'établir des bilans communaux et quelques cartographies, en particulier pour les cyclones majeurs de 1738, 1740, 1865, 1899 et 1928 . Ces bilans humains varient 
fortement suivant les sources consultées et les auteurs. Pour le cyclone de 1928 par exemple, pas moins de onze sources primaires différentes donnent des bilans allant du simple au double. Ces sources multiples ont été au nombre de douze pour le cyclone de 1865 et de huit pour celui de 1899. Le travail de recoupement des bilans officiels a cependant permis d'exprimer un bilan consolidé (tableau 1, décès 2) et des marges d'incertitude, parfois étendues lorsqu'on prend en compte les bilans établis par d'autres auteurs (tableau 1, min. et max.).

\section{Sources récentes (1950-2018)}

9 La seconde partie de l'étude s'est focalisée sur la période récente (1950 à 2018), au moyen d'archives locales (rapports, presse quotidienne), des bases de données préexistantes, de ressources en ligne et d'actes administratifs. La base de données Pluies extrêmes de Météo-France (2019), qui recense les événements pluvieux mémorables (au-delà de $70 \mathrm{~mm}$ de lame d'eau quotidienne maximale moyennée), la base Mouvements de terrain (2019), et la base GASPAR (2019) du ministère de l'Environnement, qui recense les arrêtés de catastrophes naturelles des communes françaises, ont permis d'enrichir le catalogue. Mais la grande majorité des informations a été extraite de la presse locale et notamment du quotidien France-Antilles, disponible aux archives départementales de Guadeloupe depuis 1964 et dont l'équivalent antérieur était le journal Antilles Matin. D'autres périodiques tels que Clartés ou Le Nouvelliste ont été consultés. Ces recherches documentaires ont ensuite été complétées et recoupées avec des témoignages recueillis auprès de la population locale. Enfin, la majorité des actes de décès a pu être récupérée auprès des services d'état civil des municipalités de Guadeloupe. Ces documents administratifs ont permis de valider et d'enrichir certaines informations (âge, sexe, profession, cause du décès). Cette partie du catalogue a été assortie d'un minimum de deux photographies par événement mortel, afin d'illustrer les circonstances et/ou l'intensité des aléas et les dommages occasionnés. Ainsi, cette seconde partie du catalogue a pu être doublée d'une base de données géo-référencées de type Système d'Information Géographique (SIG) permettant de localiser les victimes et de tirer un certain nombre d'informations statistiques sur les profils des victimes, leurs comportements, les causes et les circonstances des décès.

\section{Système d'Information Géographique sur la mortalité due aux aléas hydro-climatiques en Guadeloupe (SIG-MHC, 1950-2018)}

10 Pour ce faire, la structure originale de la BD Vict-In (Boissier, 2013) sur les victimes de crues méditerranéennes a été adaptée au contexte local. Cette base de données à référence spatiale, nommée SIG-MHC, se présente sous la forme d'un tableur où chaque ligne correspond à une victime. Elle est composée de 36 champs relatifs au profil et à l'identité de la victime (nom et prénom, sexe, âge, profession, commune de résidence, etc.), au lieu et moment du décès (date et heure du décès, commune du décès, coordonnées géographiques, etc.), aux causes et circonstances de décès (typologie des circonstances, aléa impliqué dans le décès, véhicule associé, etc.) ou encore aux caractéristiques des aléas impliqués (vitesse des vents, hauteur des vagues, pluviométrie). On y distingue les victimes dues à des aléas directs (vent, inondation, houle, mouvements de terrain), de celles provoquées par des aléas ou circonstances indirectes. Par ailleurs, quatre niveaux de précision dans le géo-référencement ont été 
distingués en fonction de nos capacités à localiser les victimes (à l'adresse, au quartier, à la commune, ou à la zone maritime). Ainsi, 101 victimes directes ou indirectes ont pu être localisées, dont 72 \% d'entre elles avec une précision bonne (quartier) à très bonne (adresse). Les photographies associées renseignent le moment de l'accident (photos de presse) et la situation actuelle (2018). Ces clichés permettent de voir si des aménagements de protection ont été apportés sur les lieux concernés, par exemple au niveau des passages à gué.

\section{Résultats}

\section{Événements significatifs}

11 Le catalogue historique répertorie 132 événements hydro-climatiques ayant impacté de manière significative la Guadeloupe et/ou les îles du Nord (Saint-Martin et SaintBarthélemy) depuis 1635. On peut y rajouter un événement de type foudre. Parmi eux, 17 ont surtout touché les îles du Nord, voire quasi exclusivement en 1819, 1823, 1837, 1960 (Donna), 1995 (Luis), 1996 (Bertha), 1997 (Erika), 1998 (Danielle), 1999 (José), 2017 (Irma). Les dates au mois près sont connues pour 125 d'entre eux, et au jour près pour 117. Les siècles les plus marqués sont le XX (41 événements), le XVIII ${ }^{e}$ (36) et le XIX ${ }^{e}$ (26). Les demi-siècles correspondants les plus touchés ont été les années 1950-1999 (33) et 1750-1799 (20). Au final, depuis 1635, on recense une moyenne de 3,5 événements significatifs par décennies. La décennie 1990 est la plus représentée avec quatorze événements. Les années qui ont connu le plus de cyclones ont été 1666, 1715, 1766, 1970, 1995, 1998, 1999 et 2017 avec au moins trois cyclones recensés par an. Les mois les plus concernés sont septembre (42), août (41) et octobre (15). Cette hiérarchie mensuelle est maintenue sur la période plus récente post-1950 (illustration 1).

\section{Bilans humains}

12 Les données de mortalité ont été significativement enrichies sur la première période et largement affinées sur la seconde période. Ce bilan minimum a été établi pour 45 événements dont un épisode de foudre ayant entrainé la mort de deux personnes en octobre 2014. Il a pu être établi à zéro pour 25 événements, et corrigé pour une cinquantaine d'événements identifiés par d'autres auteurs. Il a été par ailleurs enrichi d'une trentaine d'événements dont les bilans humains n'avaient jamais été cités par d'autres auteurs. Cela concerne en particulier les cyclones de 1713 (30 morts), 1738 (83), 1740 (124), 1776 (39), 1819 (36 à 38), 1821 (21), 1825 (400 à 600) et 1898 (28 morts). D'après nos corrections, sur les 133 événements recensés, au moins 45 d'entre eux ont provoqué des pertes humaines (tableau 1). Le bilan humain global est compris entre 2125 et 2502 morts, contre 1967 à 1973 identifiés par d'autres auteurs avant cette étude, soit une moyenne haute de 19 décès par événement significatif et de 56 décès par événement meurtrier connu. En prenant en compte les données apportées par d'autres auteurs, la fourchette maximale est comprise entre 2140 et 2994 victimes depuis 1635 . Les grands cyclones meurtriers en Guadeloupe remontent à 1713, 1738, 1740, 1825 (Santa-Anna), 1865, 1899 (The Great Bahamas Hurricane) et surtout 1928 avec le cyclone le plus meurtrier (Okeechobee) (1110 à 1227 morts). Ceux ayant affecté SaintMartin et Saint-Barthélemy remontent à 1819 et 1898. Sur la période d'après-guerre, Irma (2017) reste le dernier cyclone le plus meurtrier pour Saint-Martin avec onze 
morts. Il faut remonter à Hugo (1989, 16 morts), Inez (1966, 28 morts) et Cléo (1964, 13 morts) pour retrouver des bilans comparables en Guadeloupe.

\section{Répartition géographique des victimes}

Le travail sur archives (ANOM) a permis d'établir des bilans de victimes par commune actuelle de Guadeloupe pour cinq grands cyclones de la période ancienne $(1738,1740$, $1865,1899,1928)$. Cette reconstitution spatiale inédite permet de faire des hypothèses sur la trajectoire approximative de ces cyclones anciens (illustrations 2 et 3 ).

Illustration 2 - Répartition communale des décès liés aux cyclones de 1738, 1740, 1865 et 1899

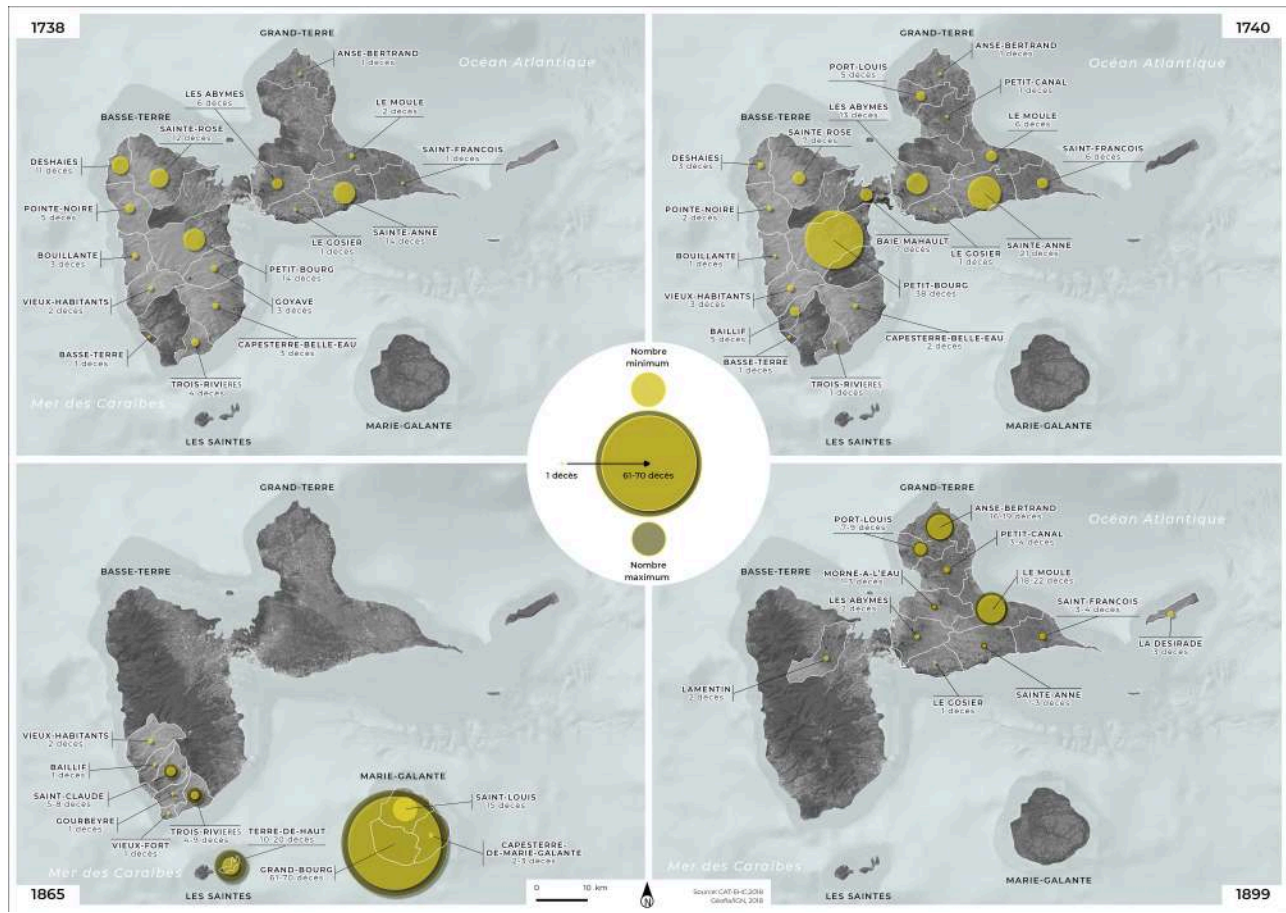

Source : CAT-EHC, 1635-2018 (UMR GRED). Auteurs : T. Candela et F. Leone.

Pour 1738, la trajectoire semble être passée au centre de la Guadeloupe, ou légèrement à l'Ouest de Grande-Terre. On peut en tirer des conclusions semblables pour 1740. Par contre, on observe une répartition symétriquement opposée des victimes de 1865 et 1899 qui semble indiquer une trajectoire sur le Sud de la Guadeloupe pour 1865 et sur le Centre/Nord de Grande-Terre pour 1899. Ces hypothèses sont corroborées par les trajectoires fournies par Fernández Partagás (2003) pour celui 1865 et par Cry (1965) pour celui de 1899 (The Great Bahamas Hurricane). Quant au cyclone de 1928, il a fortement impacté la Grande-Terre et l'Est de la Basse-Terre, ce qui suggère une trajectoire assez centrale ou légèrement portée vers l'Est, totalement en accord avec les réanalyses de trajectoires proposées par Landsea et al. (2012). Ces déductions méritent d'être recoupées avec la distribution spatiale des pertes matérielles. Elles supposent des conditions de vulnérabilité similaires à l'échelle du territoire, du moins pour le bâti. 
Illustration 3 - Répartition communale des décès liés à l'ouragan de 1928

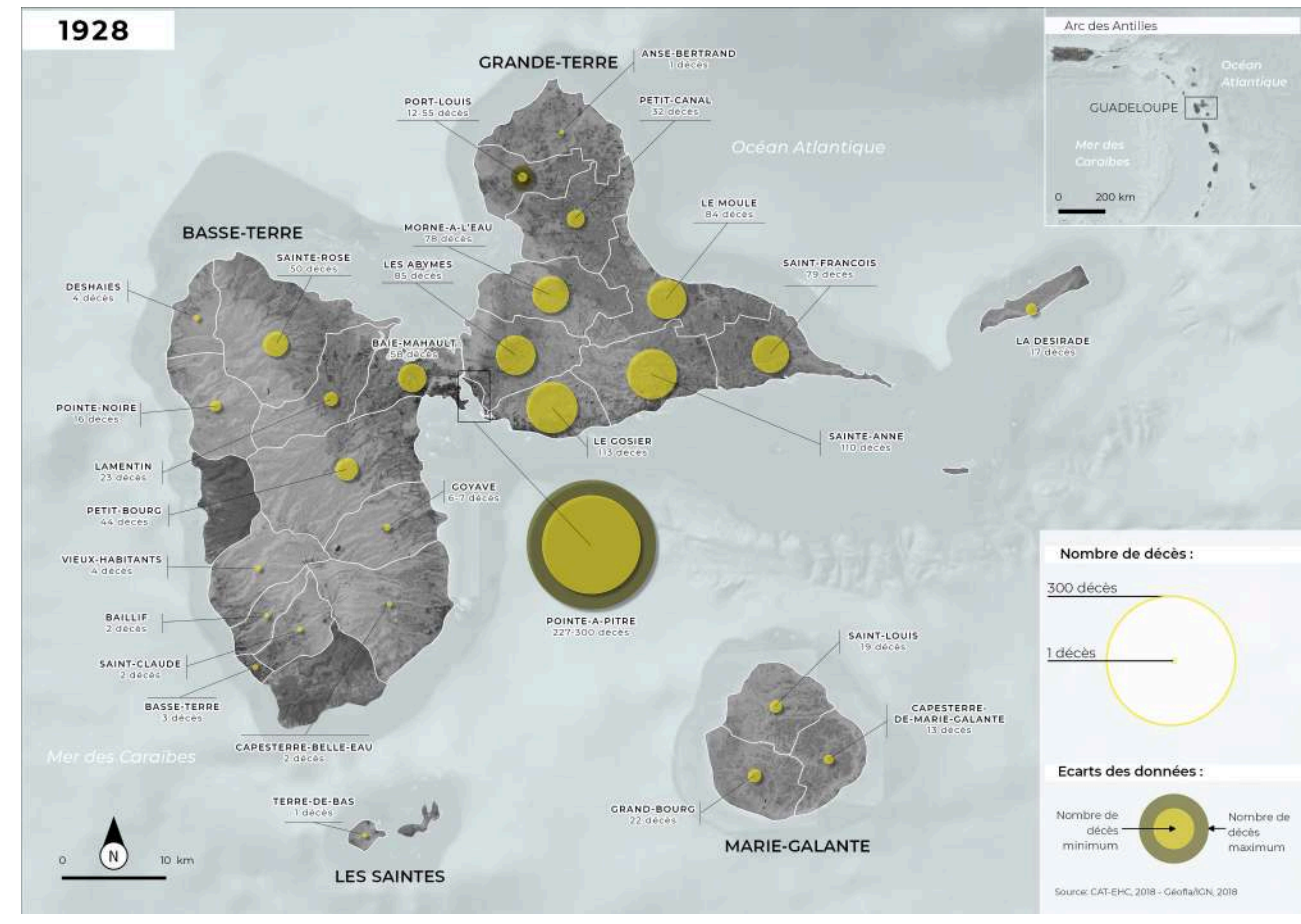

Source : CAT-EHC, 1635-2018 (UMR GRED). Auteurs : T. Candela et F. Leone.

Sur la période récente, le SIG-MHC permet d'analyser la répartition spatiale de 66 victimes directes en lien avec leur environnement immédiat (illustration 4). Les communes du Sud de Basse-Terre et du Sud de Grande-Terre enregistrent le nombre de décès le plus important. La commune de Saint-Claude est celle qui a connu le plus de décès, avec douze victimes, suivie de la commune des Abymes (11 victimes). Sur Grande-Terre, ces communes correspondent globalement aux côtes au vent, donc aux espaces les plus exposés aux effets du vent lors d'épisodes cycloniques. Mais il s'agit aussi des communes historiquement les plus peuplées donc présentant le plus d'exposition humaine (Lévy, 1963). Sur Basse-Terre, il semble exister une corrélation spatiale entre la proximité des reliefs les plus escarpés (Sud de Basse-Terre) et le nombre de morts. Le relief est un facteur important de l'occurrence des précipitations, mais aussi des glissements de terrain induits. Par ailleurs, la densité élevée du réseau hydrographique de l'île de Basse-Terre constitue sans doute un facteur d'augmentation de l'exposition aux inondations et crues soudaines. Des effets de concentration des décès lors d'un même événement sont observés. Ils correspondent à des phénomènes de "mort collective", par exemple lorsque la case familiale contenant ses huit membres est ensevelie par un glissement de terrain (quartier Papaye, Saint-Claude, cyclone Cléo, août 1964), ou bien lorsque cinq personnes sont emportées par une rivière en crue à leur domicile (quartier Marigot, Vieux-Habitant, cyclone Helena, octobre 1963), ou lors de la noyade simultanée de cinq personnes à bord d'un véhicule $4 \times 4$ au niveau d'un passage à gué (quartier Chazeau, Les Abymes, janvier 2011). 
Illustration 4 - Localisation des victimes directes issues du SIG sur la mortalité due aux aléas hydro-climatiques en Guadeloupe

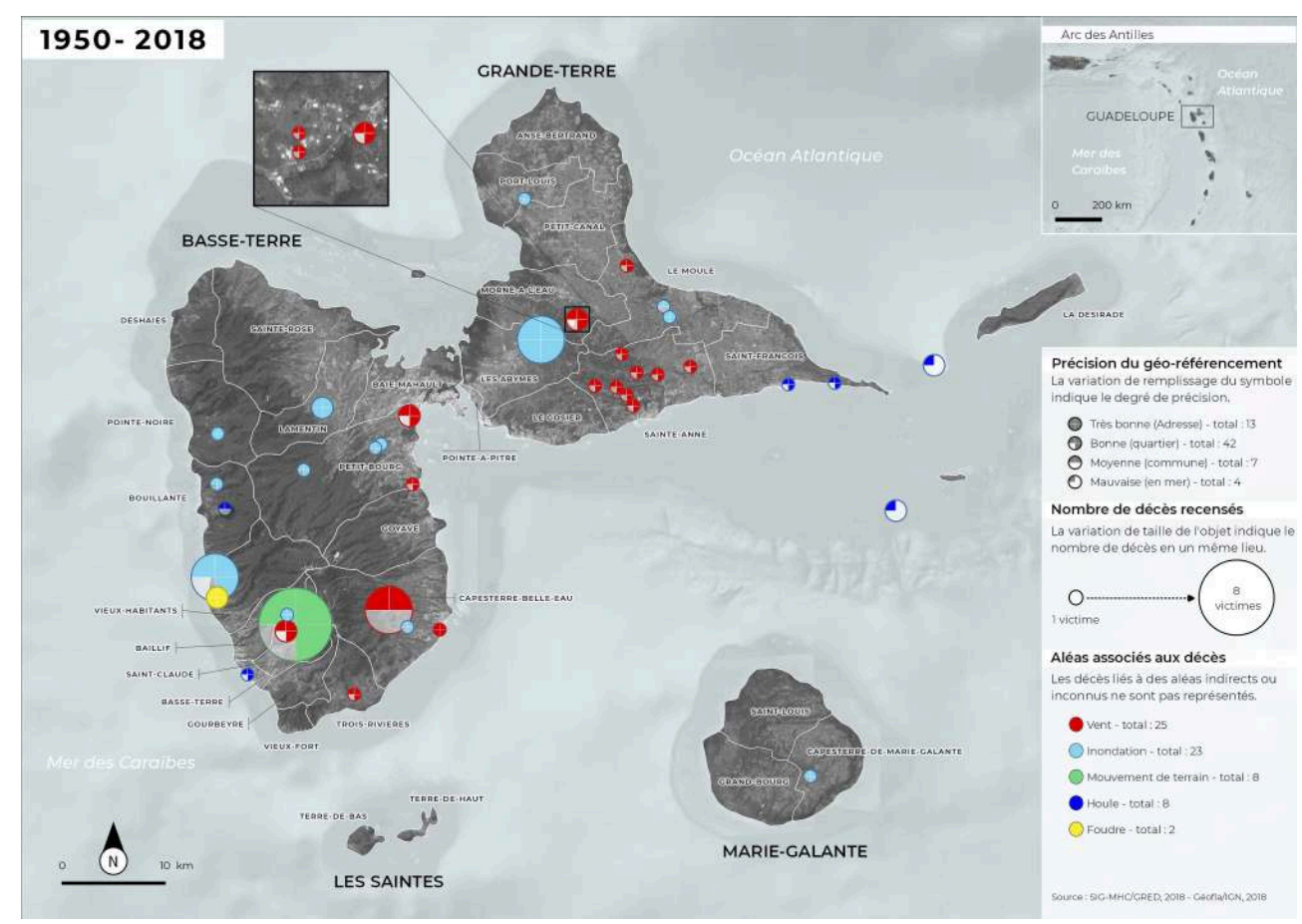

Source : SIG-MHC, 1950-2018 (UMR GRED). Auteurs : T. Candela et F. Leone.

\section{Profil des victimes}

Dans les sources historiques, la grande inconnue est bien souvent le profil des victimes. La position sociale, le sexe, les noms et prénoms, ne sont que très rarement indiqués. Outre la distinction entre citoyens libres et esclaves, nous ne savons que peu de choses. Contrairement à ce que l'on aurait pu imaginer, les morts parmi les esclaves sont assez souvent renseignés car ils sont comptabilisés dans les pertes matérielles, en vue d'une réparation. Pour cette raison, nous connaissons pour certaines dates mieux la mortalité parmi les esclaves que pour les citoyens libres. Pour le sinistre de 1738, nous avons même eu accès seulement à des états de pertes matérielles et seules les victimes nonlibres nous sont connues.

Pour la période récente, sur les 101 victimes de la BD SIG-MHC, les hommes représentent $70 \%$ de l'échantillon contre $30 \%$ de femmes. Cette surreprésentation des hommes est encore plus marquée lors des opérations de secours ou des réparations post événements ( $91 \%$ des décès). D'autre part, $75 \%$ des victimes ayant trouvé la mort dans ou suite à l'utilisation d'un véhicule lors d'un événement climatique sont des hommes. La fourchette d'âge est très large et l'âge moyen des victimes est de 37 ans, ce qui correspond à la moyenne d'âge en Guadeloupe. La structure d'âge de la mortalité liée aux événements climatiques est proche de la structure par âge de la population guadeloupéenne dans son ensemble. Parmi les personnes âgées (60 à 80 ans et plus), les personnes les plus âgées (de 70 à 80 ans et plus) s'avèrent être les plus touchées. Sur les onze victimes ayant 70 ans et plus, dix sont mortes dans un bâtiment en position d'exposition passive. Par ailleurs, 89 victimes de l'échantillon sont résidentes en Guadeloupe $(94 \%)$ et $74(78 \%)$ sont décédées dans leur commune de résidence 
permanente. Seules six victimes ( $7 \%)$ sont originaires d'une autre région française ou d'un autre pays. Il s'agit de touristes métropolitains (2) ou de militaires en déplacement dans le cadre d'une mission (2). Les deux victimes étrangères sont des plaisanciers du Guyana morts en mer pendant le cyclone Maria. Ces résultats remettent en cause le postulat selon lequel les populations auraient une meilleure conscience du risque et des comportements à tenir sur un territoire qu'elles connaissent (Blesius, 2013).

\section{Les causes et circonstances de décès}

Les circonstances de décès renseignent l'exposition des victimes, mais aussi les comportements adoptés, caractérisés ou non par une prise de risque. Parmi les 101 décès récents, les circonstances sont connues pour 89 d'entre eux. Quarante personnes ont trouvé la mort dans un bâtiment, le plus souvent leur domicile, dont 32 qui ont péri à cause de l'endommagement du bâtiment suite à la projection d'un objet ou d'un élément de façade, ou qui ont été ensevelies avec le bâtiment lors d'un glissement de terrain ou de rafales. On constate que la quasi-totalité de ces décès au domicile a eu lieu avant 1967 (97\%), ce qui reflète l'inadaptation du bâti face à l'intensité des aléas à l'œuvre sur le territoire guadeloupéen. Une circonstance de décès ressort de cette analyse avec le cas de piétons (13 victimes) emportés par les eaux alors qu'ils se livraient à des activités de loisir à proximité d'un cours d'eau (9) ou de la mer (4) lors d'événements de faible intensité, localisés et à caractère soudain (rupture d'embâcle, houle liée à un vent modéré). Ils sont pour moitié liés à une sous-estimation du risque (circulation en extérieur lors de conditions météorologiques dégradées). En ce qui concerne les victimes liées à l'utilisation d'un véhicule, une part significative $(37,5 \%)$ concerne la traversée de passages à gué inondés, avec pour moitié des véhicules tout-terrain $(4 \times 4)$. Enfin, les neuf victimes décédées lors d'une opération de sauvetage sont celles du crash de l'hélicoptère Puma survenu le 20 septembre 1989, quelques jours après le passage du cyclone Hugo. Bien que l'accident soit dû à une défaillance technique, ces victimes indirectes sont prises en compte puisque un lien entre le cyclone et les décès est établi. D'autres victimes indirectes (3) sont liées à des effets induits, différés de la manifestation des aléas. Il s'agit de victimes d'infrastructures électriques défaillantes ou liées à la réparation « autonome » du bâti endommagé.

\section{Les aléas naturels impliqués}

Les aléas impliqués sont multiples, ce qui complexifie l'analyse de l'exposition des victimes. Parmi les 101 décès récents, le phénomène naturel impliqué est connu pour 66 d'entre eux (illustration 4). Parmi les 35 décès restants, quinze sont des décès indirects, et pour les 20 derniers l'aléa responsable n'est pas renseigné. Parmi les quatre aléas de type climatique identifiés, le vent est le plus meurtrier, avec 25 victimes. Il est suivi de près par les inondations qui ont causé la mort de 23 personnes. À part égale, nous retrouvons ensuite l'aléa mouvements de terrain et la houle, ayant fait chacun huit victimes. Enfin, l'aléa foudre est à l'origine de deux victimes. La part des victimes liées au vent varie en fonction des différents événements. Elle oscille entre $16,5 \%$ et $44,5 \%$ pour les cyclones Cléo, Inès, Hugo et Maria, mais atteint $100 \%$ pour le cyclone Betsy de 1956 . Pour $96 \%$ des victimes dues au vent, les vents maximums soutenus enregistrés le jour du décès dépassent les $180 \mathrm{~km} / \mathrm{h}$. Par 
ailleurs, 31 décès sont en lien direct avec l'intensité des précipitations ou les phénomènes induits par ces dernières (crues et inondations avec 23 victimes, mouvements de terrain avec huit victimes). Entre 1950 et 2018, la houle a causé la mort de huit individus en Guadeloupe. Elle est responsable en moyenne d'un décès tous les huit à neuf ans, ce qui est beaucoup moins important que les précipitations, les autres phénomènes induits (un décès tous les 2,2 ans), ou que le vent (un décès tous les 2,6 ans).

\section{Répartition temporelle des décès}

On note une différence assez nette dans la répartition des décès entre le jour et la nuit. On atteint un effectif de 47 victimes entre $6 \mathrm{~h}$ et $18 \mathrm{~h}$ (60\%) contre 31 (40\%) entre $18 \mathrm{~h}$ et 6 h. Ces résultats diffèrent nettement de la prédominance $(75 \%)$ des décès survenant le soir ou la nuit évoquée par Mooney (1983) dans son étude sur les inondations aux ÉtatsUnis. N'affichant pas de tendance nette, la répartition circadienne des décès de notre échantillon est difficile à expliquer autrement que par l'occurrence aléatoire des aléas (Jonkman et al., 2005). Quant à elle, la répartition saisonnière est directement déterminée par la saisonnalité climatique caractéristique de la zone d'étude. En effet, la saison humide (juillet à novembre) totalise 95 des 101 décès de l'échantillon (94\%). Que ce soit depuis 1635 ou depuis 1950, c'est le mois de septembre qui totalise le plus de victimes, suivi du mois d'août. C'est la saison des cyclones et les exceptions (janvier et mai) relèvent d'épisodes pluvieux localisés, responsables de cumuls de précipitations importantes, et de décès caractérisés par une prise de risque avérée.

\section{Évolution de la mortalité récente}

21 Sur une amplitude temporelle de 1950 à aujourd'hui, la répartition des bilans humains par année montre une baisse du nombre de décès au cours du temps (illustration 5). En effet, $75 \%$ (76) des décès ont eu lieu avant 1990 et un pic de mortalité apparaît dans la décennie 1960-1970. Il correspond aux deux événements les plus meurtriers de cette période (Ines et Cléo avec respectivement 28 et 13 victimes). Dans la seconde moitié de la période étudiée, les événements meurtriers sont plus dispersés, avec des bilans humains moins importants et moins liés aux cyclones. Le cyclone Hugo (1989) constitue cependant une exception depuis 1970 avec seize victimes dont neuf indirectes. On constate par ailleurs un ressaut dans le nombre de victimes dans la période contemporaine (2011-2018). Il survient après une période de calme en termes de pertes humaines (2000-2010), qui a pu abaisser la conscience du risque et favoriser l'urbanisation en zones à risques. On a observé la même tendance avant le cyclone Hugo. Il avait été précédé d'une période de calme relatif avec uniquement des événements localisés et seulement cinq victimes entre 1971 et 1988. Depuis 1950, environ une personne meurt tous les sept mois du fait d'un événement climatique en Guadeloupe. 
Illustration 5 - Mortalité depuis 1950 et évolution de la population guadeloupéenne

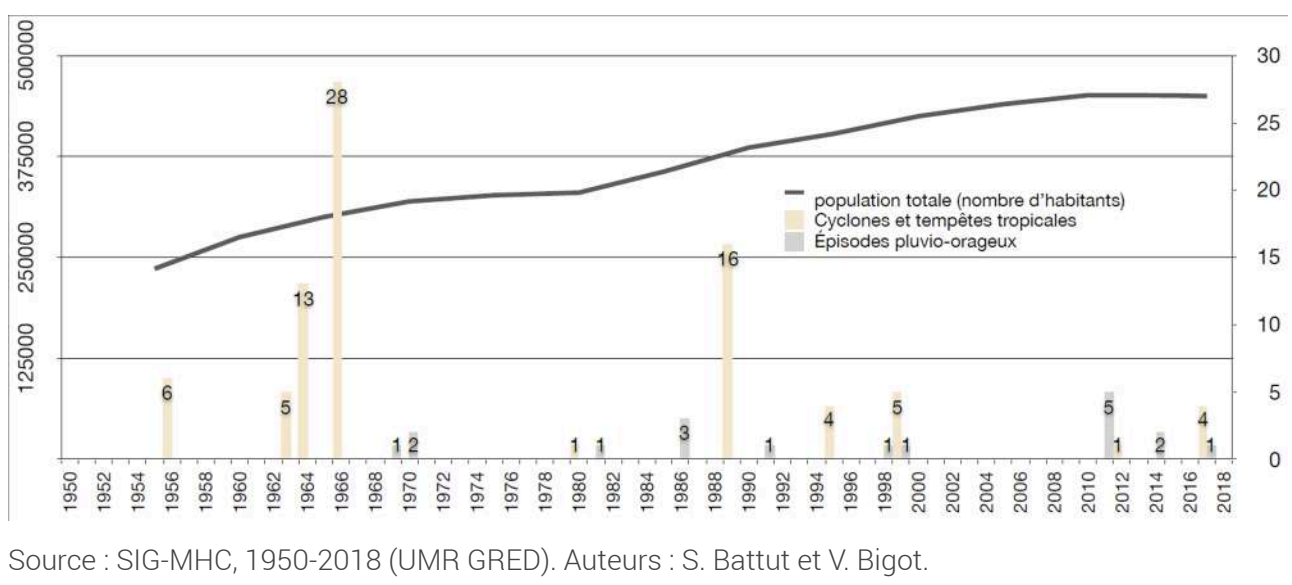

\section{La prise de risque}

La prise de risque est l'exposition volontaire aux aléas motivée par diverses raisons. Consciente ou non, elle est souvent liée à une méconnaissance ou une mauvaise estimation de la potentialité et du niveau de danger. Les comportements à risques sont donc directement déterminés par l'expérience, les représentations, individuelles ou collectives, et la perception (cognitive ou psychologique) des individus. Parmi les 101 victimes de l'échantillon récent, 28 sont décédées suite à une prise de risque ou à une sous-estimation du risque. La prise de risque la plus fréquente concerne la tentative de franchissement de passages à gué inondés à bord d'un véhicule. Ce comportement a été rapporté dans la littérature où il est souvent le fait d'hommes adultes (Boissier et Vinet, 2009). Or, dans le cas de la Guadeloupe, les conducteurs impliqués ont été en majorité des femmes de 30 à 40 ans (trois femmes contre un homme) lors d'un déplacement quotidien sur un trajet connu et à proximité du domicile. Le second type de prise de risque le plus représenté est la circulation en extérieur lors de conditions météorologiques très dégradées. Cette classe comprend des victimes circulant sur la route (3), en pleine mer (4), en bord de mer (3) lors d'un cyclone ou d'un épisode pluvio-orageux. Le franchissement ou la baignade dans une rivière en crue constituent également une situation rencontrée. Les victimes représentées avaient toutes entre 10 et 20 ans. Aucune des trois victimes n'était accompagnée par des adultes lors de l'événement et on peut estimer que c'est le manque d'expérience qui a pu jouer.

\section{Discussion : un nouveau référentiel géo-historique pour guider la prévention}

\section{Une mortalité mal renseignée par les sources historiques}

Avant 1946, la teneur des bilans de mortalité est laissée à la discrétion des gouverneurs seuls. Plus de 80 gouverneurs vont se succéder à la tête de l'île entre 1635 et 1946, et d'un mandat à l'autre, la qualité des renseignements peut varier drastiquement. Au vu des nombreux rapports que nous avons pu consulter, il est clair que la priorité pour les gouverneurs après des cyclones était davantage de trouver des solutions visant à 
assurer la subsistance des vivants plutôt qu'à renseigner le nombre de morts. Ainsi, le principal obstacle dans nos recherches a été la maigreur des données liées à la mortalité pour les événements anciens. Il n'est pas rare de lire de longs rapports qui décrivent le phénomène dans ses aspects météorologiques, relatent avec précision l'ampleur des dégâts matériels, mais occultent complètement la question des victimes. Le fait que les rapports principaux conservés aux ANOM émanent d'abord des gouverneurs de Guadeloupe, basés sur l'île principale, signifie souvent que les informations en provenance des dépendances n'arrivent pas ou plus tard. Bien souvent, les gouverneurs annoncent les morts pour la région immédiate de Basse-Terre ou de Pointe-à-Pitre et laissent le lecteur dans l'attente d'un bilan pour le reste de la colonie. Viennent ensuite les témoignages recueillis par l'administration, inclus dans des rapports sur les sinistres au côté des constats officiels des dirigeants de l'île. Ces rapports ont tendance à s'appesantir sur le sort de la population et peuvent se révéler plus instructifs. La cause principale de décès semble cependant être liée à l'effondrement de l'abri, mais cette impression peut être due au fait que les principaux témoignages viennent des villes.

Quand il est question de mortalité, se pose alors le problème de la localisation. Soit parce que les témoignages ne mentionnent pas le lieu exact des victimes, soit parce que les lieux indiqués sont incertains ou inconnus. La toponymie, justement, a aussi présenté une difficulté pour les sources les plus anciennes. Certains lieux ont changé de noms, se sont fait absorber dans des ensembles communaux, ou ont tout simplement disparu. Il nous faut aussi mentionner les sinistres pour lesquels nous ne trouvons pas de données conséquentes, mais pour lesquels d'autres chercheurs avancent pourtant des bilans. Par exemple d'après le National Hurricane Center (Rappaport et FernandezPartagas, 1997), le sinistre du 6 septembre 1776 aurait fait au moins 6000 morts pour la seule ville de Pointe-à-Pitre. Nous ne trouvons aucune source aux ANOM qui valide ce bilan. Au contraire, le gouverneur de l'époque, présent dans la ville, déclare " Je ne puis rien dire sur les pertes de Basse-Terre, il en a péri 25 à la pointe à Pitre ».

\section{Des résultats utiles pour guider la prévention}

L'approche de la vulnérabilité par la mortalité peut contribuer à une amélioration de la prévention en mettant en évidence les décès qui auraient pu être évités et qui pourront l'être en agissant sur différents leviers de cette prévention. Ainsi, parmi les 101 victimes recensées depuis 1950, nous avons pu associer à 69 d'entre elles un levier de prévention grâce auquel le décès aurait pu être évité (tableau 2). En réponse aux circonstances de décès et de prises de risques, ce sont logiquement les mesures relatives à la réduction de la vulnérabilité du bâti et à l'information préventive qui se distinguent. Quelques pistes de prévention sont discutées ci-dessous. 
Tableau 2 - Principaux leviers de prévention et nombre de victimes associées potentiellement évitables en Guadeloupe

\begin{tabular}{|c|c|c|c|c|}
\hline \multirow[t]{2}{*}{ Leviers de prévention } & \multicolumn{4}{|c|}{ Nombre de décès potentiellement évitables } \\
\hline & $1950-1976$ & 1977-1996 & 1997-2018 & Total \\
\hline Maîtrise de l'occupation des sols (PPRn) & 11 & 0 & 0 & 11 \\
\hline Adaptation du bâti & 20 & 1 & 0 & 21 \\
\hline Adaptation des comportements & 6 & 6 & 16 & 28 \\
\hline Gestion des passages à gué & 1 & 2 & 6 & 9 \\
\hline
\end{tabular}

Source : leviers de prévention d'après Boissier, 2013.

\section{Réduction de la vulnérabilité du bâti}

Parmi les 31 victimes associées à une vulnérabilité du bâti, on distingue d'une part celles reliées à une inadaptation du bâti (21) et d'autre part celles associées à une occupation des sols en zone à risque (11). Les matériaux de construction utilisés se sont avérés pas suffisamment résistants au vent et la fragilité des structures a conduit à l'arrachement du bâti par le vent ou les eaux. Dans une optique de réduction des décès liés au bâti, des normes de construction "paracycloniques » doivent être généralisées. Le passage de l'ouragan Irma sur Saint-Martin et Saint-Barthélemy en septembre 2017, tend à impulser cette mise aux normes avec la parution d'un "Guide de bonnes pratiques pour la construction et la réhabilitation de l'habitat» (CSTB, 2018) et l'adoption de nouvelles règles d'urbanisme (Collectivité de Saint-Martin, 2018). Ce renforcement progressif du bâti face aux cyclones a montré son efficacité en Guadeloupe comme l'atteste la diminution significative de la mortalité cyclonique au cours des siècles. Si la case traditionnelle en bois, souvent impliquée dans les pertes en vie humaines du passé, tend à disparaître, une grande partie du parc immobilier de Guadeloupe est située en zone de submersion marine et cumule encore de nos jours un grand nombre de critères de vulnérabilité. Un diagnostic récent effectué par notre équipe sur près de 2100 bâtiments exposés montre que $70 \%$ de ces constructions littorales ne possèdent pas d'étage refuge (illustration 6), configuration qui peut largement compromettre une mise en sécurité de leurs occupants, surtout de nuit (Vinet et al., 2011 et 2012). 

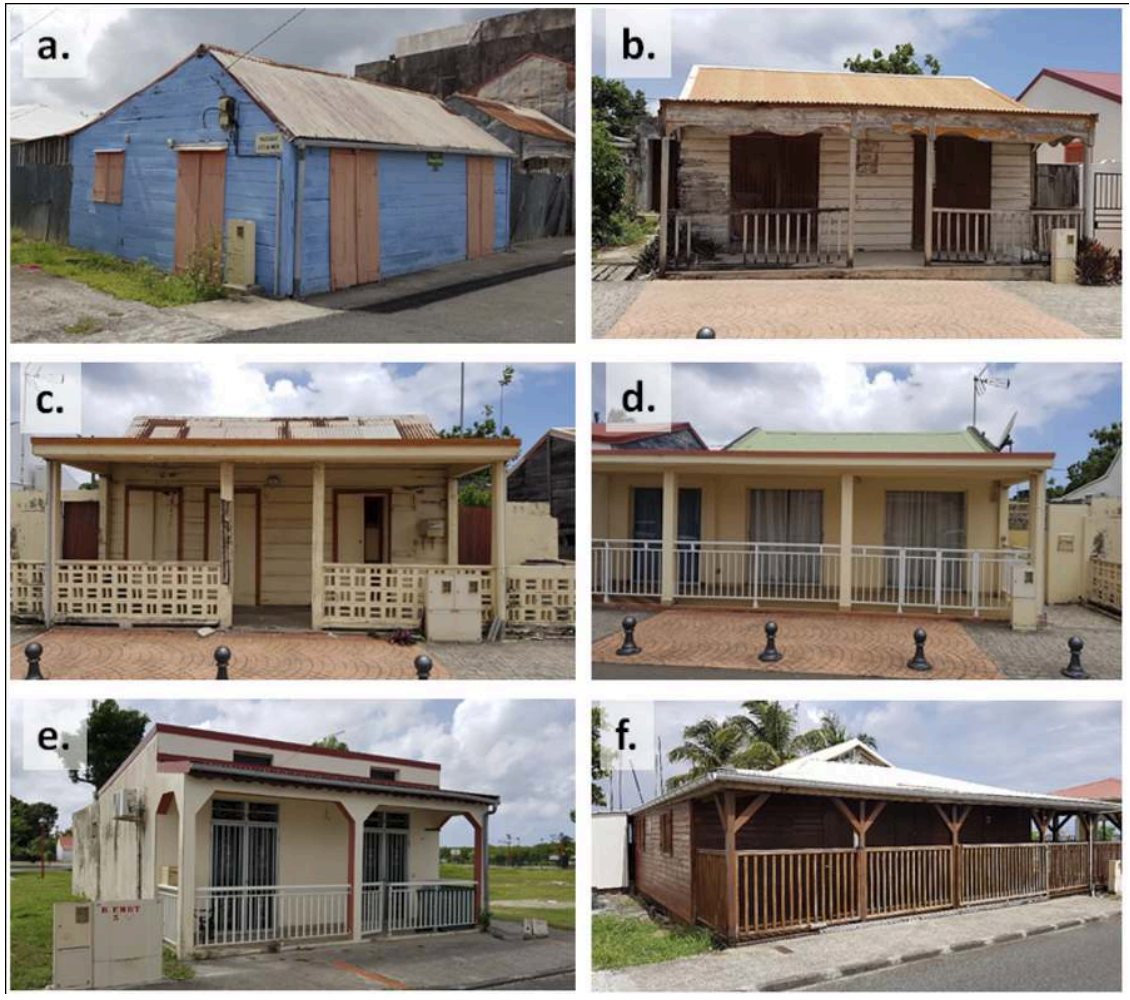

On notera les évolutions architecturales inspirées de la case traditionnelle (a) à travers les exemples b, $c$, d situés sur une même rue de Grand-Bourg à Marie-Galante.

Auteur : F. Leone, 2017.

\section{Régulation de l'occupation du sol}

Par ailleurs, 11 décès sont imputables à l'absence de maitrise de l'occupation du sol en zone à risque. Parmi elles, huit sont mortes des suites d'un glissement de terrain ayant emporté leur habitation. On note également les décès de trois personnes survenus dans des habitations situées en zones inondables. Cependant, les décès reliés à ce type d'exposition ont diminué considérablement des années 50 à nos jours. En effet, parmi les 31 victimes concernées, 30 sont décédées avant 1976 et aucune victime n'est à déplorer depuis 1997. L'adoption de PPRn depuis les années 2000 en Guadeloupe permet de réguler l'exposition aux risques naturels et de favoriser une adaptation du bâti. Néanmoins, actuellement ce sont encore $6 \%$ de la population résidente qui vivent en zones de submersion marine et un peu plus de $9 \%$ en zone inondable des PPR en vigueur ${ }^{1}$.

\section{La gestion des passages à gué}

La gestion des passages semble être un élément clé pour la réduction des bilans humains, d'autant plus que les décès liés à ce type de prise de risque ne cessent d'augmenter avec le temps. Plusieurs propositions d'amélioration de la gestion de ces passages à gué s'imposent. Dans un premier temps, un inventaire de l'ensemble des passages sur le territoire guadeloupéen est nécessaire afin de cibler les gués les plus dangereux. Ce travail a été en partie effectué dans un précédent projet visant à évaluer 
l'accessibilité routière en zone volcanique dans le Sud de Basse-Terre (Leone et al., 2018). Dans un second temps, Boissier (2013) propose d'équiper les passages les plus dangereux de barrières fixes infranchissables, expliquant que les barrières amovibles et les panneaux de signalisation ne sont pas assez dissuasifs. En janvier 2011, cinq personnes ont perdu la vie à bord de leur $4 \mathrm{x} 4$ alors qu'elles tentaient de traverser le passage à gué de Chazeau/Doubs aux Abymes. Aucune barrière amovible interdisant la traversée du gué en cas de débordement n'était présente. D'autres franchissements ne possèdent aucun équipement, comme par exemple au niveau de la Ravine Chaude sur la commune du Lamentin où une femme et son enfant sont décédés en 1986, leur voiture ayant été emportée par les eaux de la Grande Rivière Goyave en crue. De plus, l'installation de repères de crue en bordure de route et à l'entrée des ponts pourrait dissuader la traversée en cas de submersion. Boissier (2013) propose d'améliorer les règles graduées existantes en y associant un code couleur indiquant le niveau de danger.

\section{Renforcement de la culture du risque et de l'alerte}

La prise de risque et l'inadaptation des comportements ont été la cause de 28 décès entre 1950 et 2018. Cependant, ces décès ne diminuent pas avec le temps, au contraire. Au nombre de six entre 1950 et 1976, ils passent à seize entre 1997 et 2018. Onze morts étaient des piétons, onze autres ont été victimes d'un accident à bord d'un véhicule, dont neuf qui ont pris le risque de traverser un passage à gué submergé. Une personne est décédée alors qu'elle tentait de porter secours à une personne en danger et la dernière en voulant mettre ses biens en sécurité. Quatre ont été victimes d'un naufrage en mer. Quand bien même les consignes de sécurité sont comprises et appliquées par la population, encore faut-il que les gens sachent à quel moment les mettre en œuvre. C'est en ce sens qu'une amélioration des moyens d'alertes va de pair avec l'amélioration de la diffusion des consignes. En effet, une bonne communication des messages d'alerte est cruciale et peut être déterminante. Si aujourd'hui les systèmes d'alerte sont plutôt performants, notamment lorsqu'ils combinent plusieurs vecteurs: messages téléphoniques, messages radiodiffusés et télévisés, etc. Le développement des technologies smartphones apparaît comme complémentaire des dispositifs d'alertes institutionnels (Bopp et al., 2018). L'initiative récente prise par la chaine publique Guadeloupe la $1^{\text {ìre }}$ avec son portail Alerte Guadeloupe ${ }^{2}$ consacré aux risques majeurs et à leur prévention doit permettre de renforcer cette culture du risque et de la vigilance.

\section{Conclusion}

Le nouveau catalogue historique fait état de 133 événements climatiques significatifs pour la Guadeloupe et ses dépendances, dont 45 avec des victimes confirmées. Le travail sur archives a permis de consolider quelques bilans historiques, d'exhumer des événements jusqu'alors inconnus et de spatialiser des pertes humaines à l'échelle communale. Ce travail doit se poursuivre à la faveur de nouvelles investigations historiques et de recoupements complémentaires, en exploitant en particulier les registres paroissiaux. Par ailleurs, la base de données spatialisée sur les victimes a permis une approche innovante de la vulnérabilité humaine analysée par le prisme de la mortalité en lien avec les caractéristiques du territoire guadeloupéen, en mobilisant une approche géo-historique et appliquée à la prévention des risques. En Guadeloupe et 
à Saint-Martin, 112 décès ont été recensés sur la période comprise entre 1950-2018, ce qui représente 25 événements mortels décomposés en treize cyclones/tempêtes tropicales et douze épisodes pluvio-orageux. L'analyse de l'échantillon a mis en évidence, comme beaucoup d'études similaires, une surreprésentation masculine très marquée. En dépit de l'augmentation progressive des enjeux en Guadeloupe on observe une baisse significative de la mortalité au cours du temps (illustration 5). Les améliorations apportées en termes de prévention réglementaire et l'adaptation du bâti sont des facteurs indéniablement liés à cette évolution (illustration 17a).

Illustration 7 - Évolution des circonstances principales des décès (a) et des comportements à risque (b) depuis 1950
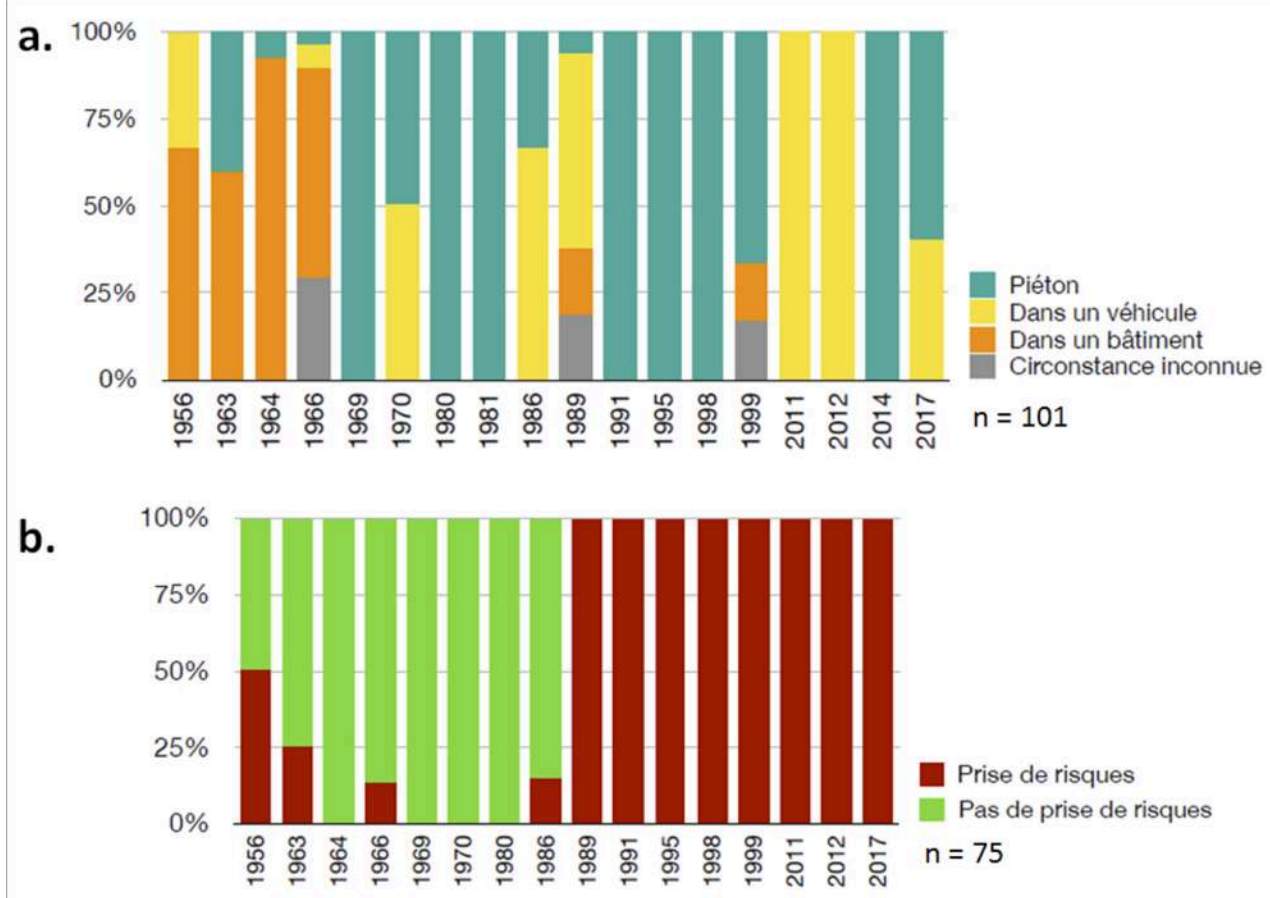

Source : SIG-MHC, 1950-2018 (UMR GRED). Auteurs : S. Battut. et V. Bigot.

Le très faible bilan humain de l'ouragan Irma sur Saint-Martin le 6 septembre 2017 témoigne d'une certaine efficacité de la prévention des risques cycloniques aux Antilles françaises, et ce malgré l'intensité des dommages liés au vent ou à la mer (Rey et al., 2019). On constate cependant que les décès sont de moins en moins liés à l'occurrence d'un cyclone, mais qu'ils sont de plus en plus associés à des comportements de mise en danger lors d'événements de faible à moyenne intensité (illustration $7 \mathrm{~b}$ ). L'éducation et la préparation des populations, adaptées au contexte local, apparaissent comme le moyen le plus efficace pour réduire ces comportements à risque. 


\section{BIBLIOGRAPHIE}

Amicale des ouragans, 2019. Atlas des cyclones de la Guadeloupe. URL : http://amicale-desouragans.org/atlas_guadeloupe/

BD ANENA (Association Nationale pour l'Étude de la Neige et des Avalanches), 2019. Bilan des accidents [En ligne]. http://www.anena.org/5041-bilan-des-accidents.htm

BD CATNAT (CATastrophes NATurelles), 2019. Observatoire permanent des catastrophes naturelles et des risques naturels [En ligne]. URL : https://www.catnat.net/

BD EM-DAT, 2019. The International Disasters Database [site web]. Brussels. OFDA/CRED, Université Catholique de Louvain. https://www.emdat.be/

BD GASPAR, 2019. Base nationale de Gestion ASsistée des Procédures Administratives relatives aux Risques [site web]. URL : http://www.georisques.gouv.fr/dossiers/telechargement/gaspar BD HI, 2019. La Base de Données Historiques sur les Inondations [site web]. URL : http://bdhi.fr/ BE Infoclimat, 2019. Infoclimat, la météo en France en temps réel - Weather in Realtime [site web]. Europe, Canada. URL : https://www.infoclimat.fr/

BD Mouvements de terrain, 2019. Base de données nationale mouvements de terrain [site web]. URL: http://www.georisques.gouv.fr/dossiers/mouvements-de-terrain\#/

BD RTM (Restauration des Terrains de Montage), 2019. Base de Données RTM [site web]. URL : https://rtm-onf.ign.fr/

BD SISFrance, 2019. Relevé et caractéristiques des séismes historiques et contemporains en France [site web]. URL : http://www.sisfrance.net/

Belmadani A., Palany P., Dalphinet A. et al., 2017. Future changes in cyclonic wave climate in the North Atlantic. 2017 AGU Fall Meeting, paper NH51A-0110 [En ligne]. URL: https://agu.confex.com/ agu/fm17/meetingapp.cgi/Paper/251612

Blesius J. C. 2013. Discours sur la culture du risque, entre approches négative et positive. Vers une éducation aux risques? Géographie et cultures, n 88, p. 249-265.

Boissier L. 2013. La mortalité liée aux crues torrentielles dans le sud de la France : une approche de la vulnérabilité humaine face à l'inondation. Thèse de doctorat, Université Montpellier 3, 215 p.

Boissier L., Vinet F., 2009. Paramètres hydroclimatiques et mortalité due aux crues torrentielles. Etude dans le sud de la France. Geographia Technica, Numéro spécial, p. 79-84.

Bopp E., Douvinet J., Serre D., 2018. Faire un tri dans le monde des applis. Expérimentations sur 50 applications smartphones proposant d'alerter la population face aux risques majeurs en France. Netcom. Réseaux, communication et territoires [En ligne]. DOI: https://doi.org/10.4000/ netcom.3531

Candale K., 1999. Morphogénèse active aux effets catastrophiques durant l'hivernage 1995 à la Martinique. In Pagney F., Leone F. (dir.), Les Antilles. Terres à risques. Paris, Ed. Karthala, p. 115-132. Cantet P., Déqué M., Palany P., Maridet J. L., 2014. The importance of using a high-resolution model to study the climate change on small islands: The Lesser Antilles case. Tellus [En ligne], vol. 66. DOI: https://doi.org/10.3402/tellusa.v66.24065 
Caviedes C. N., 1991. Five hundred years of hurricanes in the Carribean: their relationship with global 10 climate variabilities. GeoJournal, n² 23, p. 301-310.

Chenoweth M., 2006. A reassessment of historical Atlantic basin tropical cyclone activity, 1700-1855. Climatic Change, $\mathrm{n}^{\circ} 76$, p. 169-240.

Chenoweth M., Divine D., 2008. A document-based 318-year record of tropical cyclones in the Lesser Antilles, 1690-2007. Geochemistry Geophysics Geosystems [En ligne], n 9, n 8, p. 1-21. DOI: https://doi.org/10.1029/2008GC002066

Chenoweth M., Divine D., 2012. Tropical cyclones in the Lesser Antilles: descriptive statistics and historical variability in cyclone energy, 1638-2009. Climatic Change, $\mathrm{n}^{\circ} 113$, p. 583-598.

Coates. L., 1999. Flood Fatalities in Australia, 1788-1996. Australian Geographer, vol. 30, $\mathrm{n}^{\circ} 3$, p. 391-408.

Coates L., Haynes K., O’Brien J. et al., 2014. Exploring 167 years of vulnerability: An examination of extreme heat events in Australia 1844-2010. Environmental Science \& Policy, nº 42, p. 33-44.

Collectivité de Saint-Martin, 2018. Aménagement du territoire et urbanisme. Tableau récapitulatif des règles d'urbanisme et protocole, volet 2. Gouvernement 21 novembre 2017 [En ligne]. URL : http:// www.com-saint-martin.fr/ressources-Saint-Martin-Antilles.html?categorie=152

Cry G. W., 1965.

Tropical Cyclones of the North Atlantic Ocean Tracks and Frequencies of Hurricanes and Tropical Storms

1871-1963

[En ligne].United States Weather Bureau, Technical Paper 55, 66 p. https://www.nws.noaa.gov/ oh/hdsc/Technical_papers/TP55.pdf

CSTB, 2018. Guide de bonnes pratiques pour la construction et la réhabilitation de l'habitat [En ligne]. 166 p. URL : http://www.com-saint-martin.fr/ressources/

DEAL Guadeloupe, 2008. Atlas des zones inondables des cours d'eau de la Basse-Terre par des techniques d'analyse hydrogéomorphologique. Rapport, Annexe 1. Liste des inondations significatives du passé recensées en Guadeloupe et leurs principales caractéristiques, 138 p.

Defossez S., Gherardi M., Cargnelutti L. et al., (2018). Indicateurs de vulnérabilité du bâti de Guadeloupe face à la submersion marine et aux inondations. Relevés de terrain effectués sur la période juillet 2017 à mars 2018. Rapport projet PO FEDER Guadeloupe C3AF, WP5, UMR GRED, Montpellier, $44 \mathrm{p}$.

Desarthe J., 2014. Ouragans et submersions dans les Antilles françaises (XVII ${ }^{\mathrm{e}}-\mathrm{XX}{ }^{\mathrm{e}}$ siècle). Études caribéennes [En ligne], $\mathrm{n}^{\circ} 29$. URL : https://journals.openedition.org/etudescaribeennes/7176

Desarthe J., Moncoulon D., 2017. Quatre siècles de cyclones tropicaux dans les départements d'outre-mer. La météorologie, $\mathrm{n}^{\circ}$ 99, p. 52.

Fernandez-Partagas J., Diaz H. F., 1996. Atlantic Hurricanes in the second half of the Nineteenth Century. Bull. Amer. Meteor. Soc., n 77, p. 2899-2906.

French J., Von Allmen S., Wood R., 1983. Mortality from Flash Floods: A Review of National Weather Service Reports, 1969-81. Public Health Reports, vol. 98, nº 6, p. 584-588.

Garcia-Herrera R., Gimeno L., Ribera P., Hernandez E., 2005. New records of Atlantic hurricanes from Spanish documentary sources, Journal of Geophysical Research Atmospheres [En ligne], vol. 110, D03109. DOI: https://doi.org/10.1029/2004JD005272

Garnier E., Desarthe J., Moncoulon D., 2015. The historic reality of the cyclonic variability in French Antilles, 1635-2007. Climate of the Past Discussions, vol. 11, n 2, p 1519-1550. 
Guha-Sapir D., Checchi F., 2018. Editorials. Science and politics of disaster death tolls. BMJ [En ligne], 362, k4005. DOI: https://doi.org/10.1136/bmj.k4005

Hamparian R., 1999. L'ouragan Hugo sur la Guadeloupe : les enseignements d'une cartographie des effets locaux. In Pagney F., Leone F. (dir.), Les Antilles. Terres à risques. Paris, Ed. Karthala, p. 19-41.

Huc J. C., Etna M., 2015. Eclat de temps : anthologie des événements climatiques extrêmes de la Guadeloupe. PLB éditions, $456 \mathrm{p}$.

Imbert D., Labbe P., Rousteau A., 1996. Hurricane damage and forest structure in Guadeloupe, French West Indies. Journal of Tropical Ecology, vol. 12, n 5, p. 663-680.

Jonkman S. N., 2005. Global perspectives on loss of human life caused by floods. Natural hazards, vol. $34, n^{\circ}$ 2, p. 151-175.

Jonkman S. N., Kelman I., 2005. An analysis of the causes and circumstances of flood disasters deaths. Disasters, vol. 29, n 1, p. 75-97.

Jonkman S. N., Maaskant B., Boyd E., Levitan M. L., 2009. Loss of life caused by the flooding of New Orleans after Hurricane Katrina: analysis of the relationship between flood characteristics and mortality. Risk Analysis: An International Journal, vol. 29, n 5, p. 676-698.

Kishore N., Marqués D., Mahmud A. et al., 2018. Mortality in Puerto Rico after Hurricane Maria. New England journal of medicine, vol. 379, $\mathrm{n}^{\circ}$ 2, p. 162-170.

Knapp K. R., Kruk M. C., Levinson D. H. et al., 2010. The International Best Track Archive for Climate Stewardship (IBTrACS): Unifying tropical cyclone best track data. Bulletin of the American Meteorological Society, $\mathrm{n}^{\circ}$ 91, p. 363-376.

Landsea C. W., Anderson C., Charles N. et al., 2004. The Atlantic hurricane database re-analysis project: Documentation for the 1851-1910 alterations and additions to the HURDAT database. In Murnane R. J., Liu K.-B. (ed.), Hurricanes and Typhoons: Past, Present, and Future. Columbia University Press, p. 177-221.

Landsea C. W., Feuer S., Hagen A. et al., 2012. A reanalysis of the 1921-30 Atlantic hurricane database. Journal of Climate, vol. 25, $\mathrm{n}^{\circ}$ 3, p. 865-885.

Leone F., Komorowski J. C., Gherardi-Leone M. et al., 2018. Accessibilité territoriale et gestion de crise volcanique aux Antilles françaises (Guadeloupe \& Martinique) : contribution à la planification des évacuations. Cybergeo: European Journal of Geography [En ligne], document 865. DOI: https://doi.org/0510.4000/cybergeo.29425

Lévy C., 1963. Peuplement et population de la Guadeloupe. Population, vol. 18, n 1, p. 137-141.

Météo France, 2019. Pluies extrêmes en France métropolitaine et en outre-mer [En ligne]. URL : http:// pluiesextremes.meteo.fr/

Mooney L. E., 1983. Applications and implications of fatality statistics to the flash flood problem. Preprints. In The American Meteorological Society Fifth Conference on Hydrometeorology.

Nicolas T., Pagney Bénito-Espinal F., Lagahé E., Gobinddass M. L., 2018. Les catastrophes cycloniques de septembre 2017 dans la Caraïbe insulaire au prisme de la pauvreté et des fragilités sociétales. EchoGéo [En ligne], n 46. DOI: https://doi.org/10.4000/echogeo.16439

Pagney F., 1991. Genèse et dynamique de l'ouragan Hugo sur la Guadeloupe. Annales de géographie vol. $100, \mathrm{n}^{\circ} 558$, p. 152-165. 
Pagney F., 1998. Trois ouragans sur la Guadeloupe : Hugo (1989), Luis et Marilyn (1995) et l'activité touristique. In Catastrophes naturelles aux Antilles. D'une Soufrière à une autre, Paris, Ed. Karthala/CERC, p. 183-196.

Fernández Partagás J., 2003. Year 1865. National Oceanic and Atmospheric Administration [En ligne]. URL : https://www.aoml.noaa.gov/hrd/Landsea/Partagas/1865-1870/1865.pdf

Pilon R., Chauvin F., Belmadani A., Palany P., 2017. North Atlantic tropical cyclones: Historical simulations and future changes with the new high-resolution Arpege. AGCM. 2017 AGU Fall Meeting [En ligne], paper A53C-2258. URL: https://agu.confex.com/agu/fm17/meetingapp.cgi/Paper/ 239137

Poey A., 1855. A chronological table, comprising 400 cyclonic hurricanes which have occurred in the West Indies in the North Atlantic within 362 years, from 1493 to 1855. Journal of the Royal Geographical Society, n² 25, p. 291-328.

Rappaport E. N., Fernandez-Partagas J., 1997. NOAA Technical Memorandum NWS NHC 47 [En ligne]. Updated 22 April 1997 by Jack Beven. URL: https://www.nhc.noaa.gov/pastdeadly.shtml

Rappaport E. N., 2000. Loss of life in the United States associated with recent Atlantic tropical cyclones. Bulletin of the American Meteorological Society, vol. 81, n 9, p. 2065-2074.

Rey T., Leone F., Candela T. et al., 2019. Coastal Processes and Influence on Damage to Urban Structures during Hurricane Irma (St-Martin \& St-Barthélemy, French West Indies). Journal of Marine Science and Engineering [En ligne]. vol. 7, n 7, p. 215. DOI: https://doi.org/10.3390/ jmse7070215

Saffache P., Marc J. V., Huyghes-Belrose V., 2003. Les cyclones en Guadeloupe : quatre siècles cataclysmiques : éléments pour une prise de conscience de la vulnérabilité de l'île de l'archipel guadeloupéen. Ibis rouge éditions, 278 p.

Sarant P.M., de Vanssay B., Leone F. et al.,. 2003. Retour d'expérience sur la prise de décision et le jeu d'acteurs : le cas du cyclone Lenny dans les Petites Antilles au regard du passé. Rapport programme EPR, $332 \mathrm{p}$.

Vinet F., 2017. Impacts humains et sanitaires des inondations. In Vinet F. (ed), Inondations 1. La connaissance du risque. iSTE Editions, p. 49-68.

Vinet F., Boissier L., Defossez S., 2011. La mortalité comme expression de la vulnérabilité humaine face aux catastrophes naturelles : deux inondations récentes en France (Xynthia, Var, 2010). Vertigo, vol. $11, \mathrm{n}^{\circ} 2$.

Vinet F., Lumbroso D., Defossez S., Boissier L., 2012. A comparative analysis of the loss of life during two recent floods in France: the sea surge caused by the storm Xynthia and the flash flood in Var. Natural hazards, vol. 61, n 3, p. 1179-1201.

Vinet F., Boissier L., Saint-Martin C., 2016. Flashflood-related mortality in southern France: first results from a new database. 3rd European Conference on Flood Risk Management (FLOODrisk 2016), Lyon, France. E3S Web of Conferences

Zahibo N., Pelinovsky E., Talipova T. et al., 2007. Statistical analysis of cyclone hazard for Guadeloupe, Lesser Antilles. Atmospheric Research, vol. 84, n 1, p. 13-29. 


\section{NOTES}

1. Calculs issus du croisement des cartes du PPRn actuellement en vigueur de Guadeloupe (http://pprn971guadeloupe.fr/) avec la base de données MAJIC de données de population 2015 à l'échelle du bâti du Laboratoire Central de Surveillance de la Qualité de l'Air (https:// www.lcsqa.org/fr/rapport/2015/ineris/fourniture-donnees-population-spatialisees-selonmethodologie-nationale-methode-).

2. https://la1ere.francetvinfo.fr/alerte-guadeloupe?r=guadeloupe

\section{RÉSUMÉS}

Cet article pose les bases méthodologiques d'un catalogue historique des évènements hydrométéorologiques significatifs et mortels qui ont frappé l'archipel de Guadeloupe et les îles du Nord (Saint-Martin et Saint-Barthélemy) depuis 1635. Pour la période récente, post 1950, il est doublé d'un Système d'Information Géographique permettant de localiser et de renseigner chaque victime avec son profil, son comportement, les circonstances et causes du décès. Outre l'apport de bilans humains consolidés, ce nouveau référentiel géo-historique permet d'analyser l'évolution de la mortalité au cours du temps en lien avec les principaux facteurs de vulnérabilité. On peut en tirer un certain nombre d'enseignements utiles pour guider la prévention des risques en Guadeloupe.

This paper lays the methodological basis for a historical catalogue of significant and deadly hydro-meteorological events that have occurred in the Guadeloupe archipelago and the northern islands since 1635. For the recent period, post 1950, it is supplemented by a Geographical Information System used to locate and inform each victim with his profile, his behavior, the circumstances and causes of death. In addition to the contribution of consolidated human death tolls, this new geo-historical framework makes it possible to analyze the evolution of mortality over time in relation to the main factors of vulnerability. A number of useful lessons can be drawn from this to guide risk prevention in Guadeloupe.

\section{INDEX}

Keywords : mortality, vulnerability, cyclone, Guadeloupe, West Indies

Mots-clés : mortalité, vulnérabilité, cyclone, Guadeloupe, Antilles

Thèmes : Sur le Champ - Sur le Terrain

\section{AUTEURS}

\section{FRÉDÉRIC LEONE}

Leone Frédéric, Frederic.Leone@univ-montp3.fr, est professeur à l'Université Paul Valéry

Montpellier 3. Il a récemment publié :

- Rey T., Leone F., Candela T. et al., 2019. Coastal Processes and Influence on Damage to Urban Structures during Hurricane Irma (St-Martin \& St-Barthélemy, French West Indies). Journal of 
Marine Science and Engineering [En ligne]. vol. 7, n 7, p. 215. DOI: https://doi.org/10.3390/ jmse7070215

- Bennani O, Druon E., Leone F. et al., 2019. A spatial and integrated flood risk diagnosis. Relevance for disaster prevention at Ourika valley (High Atlas-Morocco). Disaster Prevention and Management [En ligne], vol. 28 n 5, p. 548-564. DOI : https://doi.org/10.1108/DPM-12-2018-0379 - Leone F., Komorowski J.C., Gherardi-Leone M. et al., 2018. Accessibilité territoriale et gestion de crise volcanique aux Antilles françaises (Guadeloupe \& Martinique) : contribution à la planification des évacuations. Cybergeo. European Journal of Geography, Espace, Société, Territoire [En ligne], document 865. http://journals.openedition.org/cybergeo/29425 - DOI: https://doi.org/ $10.4000 /$ cybergeo.29425

\section{SAMUEL BATTUT}

Samuel Battut, Battut.Samuel@gmail.com, est doctorant à l'Université Paul Valéry Montpellier 3

VICTORIA BIGOT

Victoria Bigot Victoriabigot@gmail.com, est rattachée à l'Université Paul Valéry Montpellier 3

\section{GUILHEM COUSIN THOREZ}

Guilhem Cousin Thorez, Guilhem.Cousinthorez@yahoo.fr, est rattaché à Aix-Marseille Université

\section{THOMAS CANDELA}

Thomas Candela, Candela@riscrises.com, est doctorant à l'Université Paul Valéry Montpellier 3 et responsable du pôle R\&D du BE RisCrises. Il a récemment publié :

Rey T., Leone F., Candela T. et al., 2019. Coastal Processes and Influence on Damage to Urban Structures during Hurricane Irma (St-Martin \& St-Barthélemy, French West Indies). Journal of Marine Science and Engineering. vol 7, nº 7, p. 215. DOI: https://doi.org/10.3390/jmse7070215

\section{FREDDY VINET}

Freddy Vinet, Freddy.Vinet@univ-montp3.fr, est professeur à l'Université Paul Valéry Montpellier 3. Il a récemment publié :

- Vinet F., 2018. La Grande Grippe. 1918 : la pire épidémie du siècle. Histoire de la grippe espagnole. Paris, Éd. Vendémiaire, $260 \mathrm{p}$.

- Vinet F. (dir.), 2018. Inondations 1. La connaissance du risque. Londres, Elsevier/ISTE éditions, $388 \mathrm{p}$.

- Vinet F. (dir.) 2018. Inondations 2. La gestion du risque. Londres, Elsevier/ISTE éditions, 470 p. 